\title{
SPEAR far UV spectral imaging of highly ionized emission from the North Galactic Pole region
}

\author{
B. Y. Welsh ${ }^{1}$, J. Edelstein ${ }^{1}$, E. J. Korpela ${ }^{1}$, J. Kregenow ${ }^{1}$, M. Sirk ${ }^{1}$, K.-W. Min ${ }^{2}$, J. W. Park ${ }^{2}$, \\ K. Ryu ${ }^{2}$, H. Jin ${ }^{3}$, I.-S. Yuk ${ }^{3}$, and J.-H. Park ${ }^{3}$ \\ 1 Space Sciences Laboratory, University of California, 7 Gauss Way, Berkeley, CA 94720, USA \\ e-mail: bwelsh@ssl. berkeley.edu \\ 2 Korea Advanced Institute of Science \& Technology, 305-70 Daejeon, Korea \\ 3 Korea Astronomy \& Space Science Institute, 305-348 Daejeon, Korea
}

Received 20 April 2007 / Accepted 25 May 2007

ABSTRACT

\begin{abstract}
Aims. We present far ultraviolet (FUV: 912-1750 $)$ ) spectral imaging observations recorded with the SPEAR satellite of the interstellar

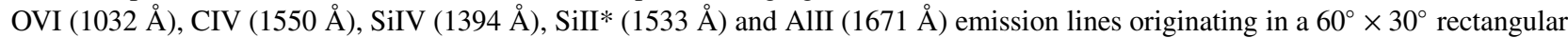
region lying close to the North Galactic Pole. These data represent the first large area, moderate spatial resolution maps of the distribution of UV spectral-line emission originating the both the highly ionized medium (HIM) and the warm ionized medium (WIM) recorded at high galactic latitudes.

Methods. By assessing and removing a local continuum level that underlies these emission line spectra, we have obtained interstellar emission intensity maps for the aforementioned lines constructed in $8^{\circ} \times 8^{\circ}$ spatial bins on the sky.

Results. Our maps of OVI, CIV, SiIV and SiII* line emission show the highest intensity levels being spatially coincident with similarly high levels of soft X-ray emission originating in the edge of the Northern Polar Spur feature. However, the distribution of the low ionization AlII emission does not show this spatial correlation, and suggests that warm-neutral and/or partially ionized gas with a temperature $<20000 \mathrm{~K}$ may be quite pervasive at high galactic latitudes.

The variation of the emission line intensity ratios as a function of sky position is contrasted with theoretical predictions concerning the physical state of interstellar gas in the galactic halo. The observed line ratios alone unfortunately do not provide us with a clear diagnostic tool to distinguish between the various physical production mechanisms responsible for both high and low ion states. However, our results do favor the hybrid model of Shull \& Slavin (1994, ApJ, 427, 784) which incorporates the combined effects of turbulent mixing layers and isobarically cooling supernova remnant gas. For this highly ionized gas, our results are best explained assuming that the observed OVI halo emission is somewhat clumpy in nature, consistent with its production at interfaces between warm $\left(T=10^{3}-10^{4} \mathrm{~K}\right)$ and hotter $\left(T=10^{6} \mathrm{~K}\right)$ soft X-ray emitting gas. CIV emission at these interfaces occurs in the intermediate temperature $\left(T=10^{5} \mathrm{~K}\right)$ gas, which seems always present whenever OVI is strongly detected. Alternately, the data are also consistent with CIV emission being ubiquitous throughout the halo with a fairly constant level of emission line intensity (of $\sim 4000$ LU), and our observations mostly reflect the superposition of spatially separate OVI emission originating at the cloud interfaces of random clumps of high latitude gas.
\end{abstract}

Key words. ISM: atoms - ultraviolet: ISM

\section{Introduction}

The emission properties of the interstellar (IS) gas and its associated spatial distribution on the sky are well known at most wavelengths except for the far ultraviolet region (912 ̊-2000 ̊). For example, emission from the cold $(T<100 \mathrm{~K})$ interstellar medium (ISM) is best observed at radio and millimeter wavelengths, particularly through $21 \mathrm{~cm}$ mapping of atomic hydrogen (Burton \& Hartmann 1994). In contrast, the emitting properties of hot IS gas with temperatures $>10^{6} \mathrm{~K}$ are best revealed through X-ray observations, as shown by the all-sky emission maps recorded by the ROSAT satellite (Snowden et al. 1997). In the visible region, all-sky maps of $\mathrm{H} \alpha$ emission have provided us with a wealth of new insights into the spatial and kinematic structure of the warm $\left(T \sim 10^{4} \mathrm{~K}\right)$ and ionized component of gas in our Galaxy (Haffner et al. 2003). However, although diffuse ultra-violet (UV) emission from some very small regions of the sky has been previously reported (Hurwitz 1994; Shelton et al. 2001; Murthy \& Sahnow 2004), no large-scale sky-survey in the UV had been attempted until the launch of the SPEAR instrument in 2003 (Edelstein et al. 2006a). SPEAR, flown aboard the
Korean STSAT-1, has now provided us with the first UV spectral imaging survey (912-1150 $\AA$ and 1350-1750 $\AA$ ) of $\sim 80 \%$ of the sky recorded at a spectral resolution of $\lambda / \Delta \lambda \sim 550$ and an imaging resolution of $\sim 5$ arcmin (Edelstein et al. 2006b)

Far UV photons ionize interstellar (HI) gas atoms, dissociate interstellar $\mathrm{H}_{2}$ molecules and provide a significant contribution to the heating of the ISM through the liberation of electrons from interstellar dust grains and by directly exciting IS atoms and molecules (Black \& van Dishoeck 1987). The far UV spectrum of diffuse interstellar emission contains the astrophysically important cooling lines of CIV (1550 ̊), SiIV (1394 A) and OVI $(1032 \AA)$ for a typical IS plasma with a temperature in the range $T=10^{4.5}-10^{5.7} \mathrm{~K}$ (Korpela et al. 2006). In addition, emission from the SiII* (1533 $\AA$ ) and AlII (1671 $\AA$ ) lines, which probe the warm $\left(T \sim 10^{4} \mathrm{~K}\right)$ and partially-ionized ISM (Jenkins \& Tripp 2001), are also prominent. Thus, spectral imaging of this diffuse far UV emission can reveal the physical conditions, history, spatial distribution, cooling physics and hydrodynamic processes of the galactic ISM. The major component to the measured far UV diffuse flux in most galactic directions is that of starlight (from 
hot OB stars), which is scattered by interstellar dust (Bowyer 1991). Thus, SPEAR spectral maps of regions in and near the galactic plane correlate well with those of reddening and HI column density and are thus complimentary to those recorded by the IRAS and COBE missions (Finkbeiner et al. 1998). However, at high latitudes in the galactic halo the diffuse far UV emission signal, although far weaker than that recorded at lower galactic latitudes, is essentially uncontaminated by the combined effects of scattered UV photon emission from dust, hot stars and interstellar molecules.

The detailed spatial distribution of the highly ionized gas in the halo, as traced by the important UV lines of CIV, SiIV and OVI, is not known in great detail since such data has been gained mainly from absorption measurements along individual sightlines to a limited number of distant OB stars and AGN. However, the recent FUSE absorption survey of OVI absorption in the halo has revealed large irregularities in the distribution of highly ionized gas that suggests a significant amount of both small- and large-scale structure in this ionized medium (Savage et al. 2003). Furthermore, in a recent survey of diffuse OVI emission along 183 sight-lines with the FUSE satellite, Dixon et al. (2006) have found that the OVI emitting regions at high galactic latitudes are intrinsically fainter than those at low latitudes, which may indicate two different populations of highly ionized emitting gas. Although most of the OVI emission line-intensities lie in the fairly restricted range of 1800 to $5500 \mathrm{LU}$ (line unit; 1 photon $\mathrm{cm}^{-2} \mathrm{~s}^{-1} \mathrm{sr}^{-1}$ at $1032 \AA$ ), the nearby local emitting OVI regions have higher electron densities and far smaller path lengths than regions emitting at high galactic latitudes.

The ions of CIV and SiIV commonly trace IS gas with a lower temperature of $\sim 10^{4.5-5.0} \mathrm{~K}$. In a study of high ion absorption through the galactic halo by Savage et al. (1997), the authors found that the column density ratio of $N(\mathrm{CIV}) / N(\mathrm{SiIV})$ was relatively constant (with a value of 4.2) for most sightlines irrespective of the galactic latitude being probed. However, there is growing evidence that the column density ratio of $N(\mathrm{CIV}) / N(\mathrm{OVI})$ in the galactic halo does not follow such behavior and its measured value along a particular halo sight-line may well reflect the local nature of ionization processes present (Zsargo et al. 2003; Savage et al. 2003). This may be expected if UV radiation from the central stars of OB associations and stellar wind-driven bubbles is escaping into the halo and producing the CIV and SiIV ions, but is not contributing to the emission from the higher ionization species of OVI and NV ions which are formed mainly by collisional ionization processes (Ito \& Ikeuchi 1988). Unfortunately studies of the important CIV and SiIV lines observed in emission have proven problematic for observers (see Bowyer 1991 for a review). Apart from the detections of diffuse emission with line intensities of 2000-5700 LU from the lines of CIV and OIII along several sight-lines by Martin \& Bowyer (1990), no other similar emission measurements had been made during the intervening years until the launch of the SPEAR instrument in 2003 (Edelstein et al. 2006a).

Thus, in order to explore the spatial distribution of $10^{4.0}-10^{6} \mathrm{~K}$ emitting gas in the halo we present SPEAR spectral imaging data of the interstellar OVI, CIV, SiIV, SiII* and AlII emission lines originating in rectangular region of size $\sim 60^{\circ} \times 30^{\circ}$ lying close to the North Galactic Pole. This high latitude region is largely devoid of bright and hot $\mathrm{OB}$ stars (that can contaminate the observed far UV emission signal), and has a relatively low value of interstellar reddening $(<0.05)$ and is located far away from the effects of scattered starlight from the underlying galactic plane regions. Our SPEAR observations of the the OVI, CIV and SiIV emission lines thus provide us with a potential probe of the the hot $\left(10^{5-6} \mathrm{~K}\right)$ and highly ionized medium (HIM), whereas the SiII* and AlII lines trace the cooler $\left(10^{4} \mathrm{~K}\right)$ gas of the warm and partially-ionized medium (WIM). These FUV data are presented in the form of spectral line image maps binned into $8^{\circ} \times 8^{\circ}$ regions, with an initial instrumental spatial resolution of $\sim 15$ arcmin.

\section{Observations and data reduction}

We present observations of diffuse emission from near the North Galactic Pole region recorded with both the long wavelength ( $L$-band: $1350-1750 \AA$ ) and short wavelength ( $S$-band: 912-1150 A) channels of the SPEAR imaging UV spectrograph flown aboard the Korean STSAT-1 satellite, which was launched in September 2003 (Edelstein et al. 2006a,b). An area of sky covering a rectangular strip of size $\sim 60^{\circ} \times 30^{\circ}$ centered on $\left(l=280^{\circ}\right.$, $\left.b=+70^{\circ}\right)$ was observed with repeated scans using the $4^{\circ} \times 4.3^{\prime}$ $S$-band and $7.5^{\circ} \times 4.3^{\prime} L$-band entrance apertures, which resulted in a total on-sky exposure time of $32 \mathrm{ks}$ obtained from $\sim 300$ orbital scans.

Full details of the SPEAR scientific mission, its instrument design and modes of on-orbit observation can be found in Edelstein et al. (2006a,b). The standard data reduction procedures for SPEAR observations are described in detail by Korpela et al. (2006) and consist of 4 main elements that include: (i) remapping of each time-tagged FUV photon to a position on the sky based on knowledge of the spacecraft's time and attitude information; (ii) correction for the effective exposure level for each sky-pixel; (iii) removal of stellar contamination from the collected photons by excluding spatial bins with $>3$ times the local median count rate; and (iv) binning the resultant data to an effective spatial resolution of $\sim 15^{\prime}$ pixels on the sky. Each of these sky-pixels can thus be associated with a data cube of information containing the FUV emission spectrum (i.e. wavelength versus counts $\mathrm{s}^{-1}$ ) summed over the area of a sky-pixel at a particular galactic position $(l, b)$ on the sky. Thus, the resultant data product from such observations is a far UV spectral image for each sky-pixel.

In Figs. 1 and 2 we show the total summed UV spectra (for the respective $S$ - and $L$-bands) from all of the sky-pixels contained within the $\sim 60^{\circ} \times 30^{\circ}$ area of the sky presently under investigation. These summed spectra, which contain contributions from areas of both intrinsically high and low FUV signal, have been binned to a spectral resolution of $1.5 \AA$ ( $S$-band) and $3.0 \AA$ ( $L$-band). As can be clearly seen in Fig. 2 , the dominant component to the measured flux at longer FUV wavelengths is from the underlying continuum, which consists of contributions from the detector background, dust-scattered continua and scattered airglow within the instrument. Although not a true continuum signal, this underlying level corresponds to $\sim 950 \mathrm{CU}$ (continuum intensity units; photons $\mathrm{s}^{-1} \mathrm{~cm}^{-2} \mathrm{sr}^{-1} \AA^{-1}$ ) at a wavelength of $1550 \AA$. At shorter wavelengths the underlying continuum contribution from scattering by dust is somewhat reduced and the dominant source of emission arises in scattering from the Lyman series of geo-coronal hydrogen lines. As a guide, this underlying signal is $\sim 3300 \mathrm{CU}$ at $1030 \AA$. However, our main interest in this paper is the overlying (far fainter) astrophysical emission lines of OVI, CIV, SiIV, SiII* and AlII formed in the diffuse ISM. We also note the low level of emission from molecular $\mathrm{H}_{2}$ fluorescence lines (at $1608 \AA$ ), which Edelstein et al. (2006a) have detected ubiquitously at galactic latitudes $<50^{\circ}$. This can be explained by the presence of very low levels of interstellar dust found at the presently observed high galactic latitudes. 

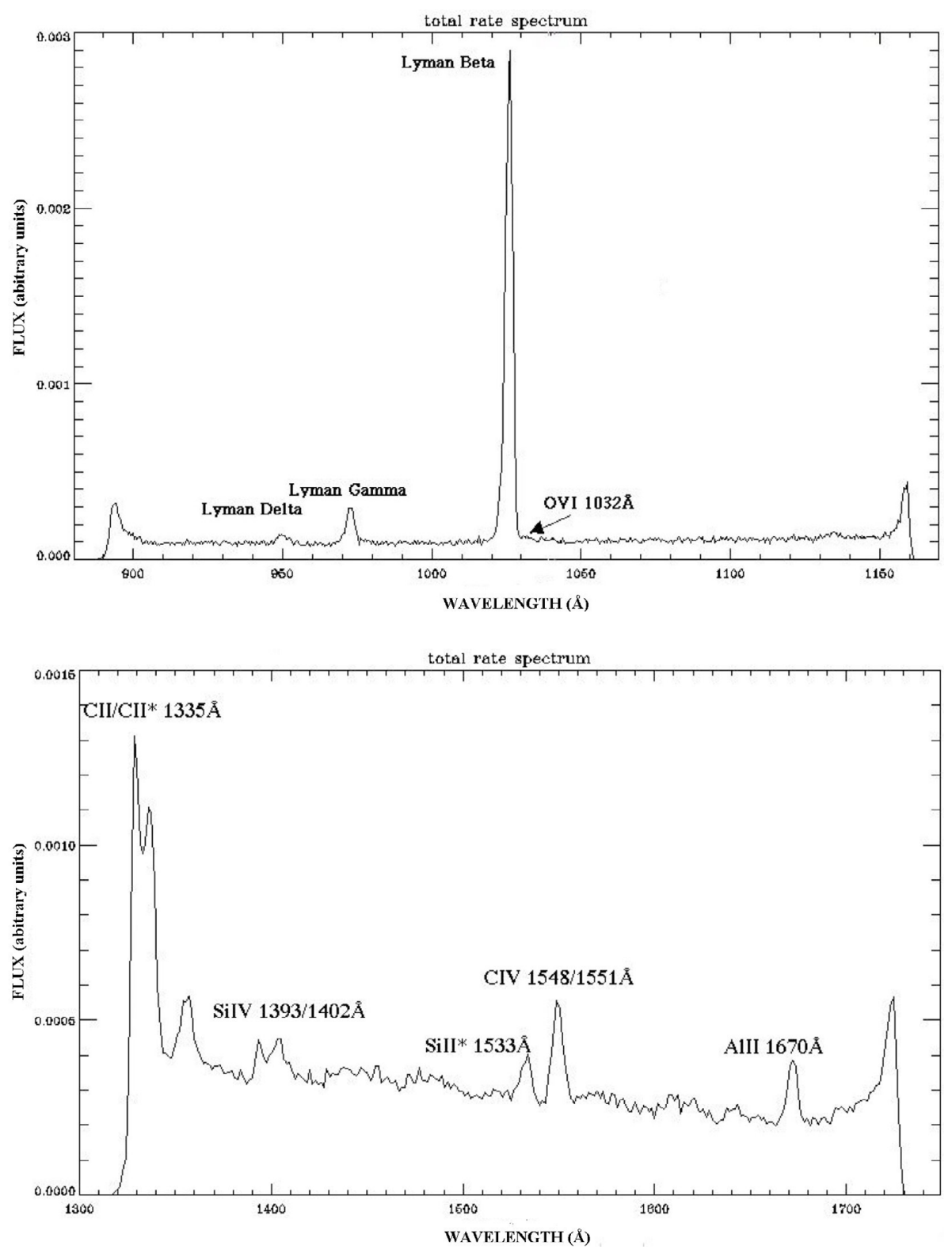

Fig. 1. Summed far UV $S$-band $(900-1150 \AA)$ SPEAR spectrum of the $\sim 60^{\circ} \times 30^{\circ}$ area of sky near to the North Galactic Pole region. The underlying "continuum" level amounts to $\sim 3300 \mathrm{CU}$ at $1030 \AA$. See Table 1 for total summed line intensity values.
Fig. 2. Summed far UV $L$-band (1350-1750 ̊) SPEAR spectrum of the $\sim 60^{\circ} \times 30^{\circ}$ area of the sky near to the North Galactic Pole region. The underlying "continuum" level is $\sim 1000 \mathrm{CU}$ at $1550 \AA$ A. See Table 1 for total summed line intensity values.
In order to improve the resultant $\mathrm{S} / \mathrm{N}$ ratio of the data, the 15 arcmin sky-pixels were binned into far larger $8^{\circ} \times 8^{\circ}$ elements to obtain a summed, exposure corrected FUV spectrum over areas of 64 sq. deg on the sky. For each of the emission lines of interest in these large sky-pixel FUV spectra, an underlying continuum level (modeled using a dust scattered population of upper main sequence stars as described in Korpela et al. 2006) was assessed over a wavelength range extending $\sim \pm 10 \AA$ from the expected central position of the spectral line. As part of this continuum assessment a best-fit Gaussian emission profile (superposed upon this level) was also attempted for any emission feature within $\pm 1.5 \AA$ (i.e. half of one SPEAR resolution element) from the theoretical line center wavelength. This is an iterative process that seeks to simultaneously best-fit both the underlying continuum level and the overlying Gaussian profile, such that the result is a residual intensity (in counts $\mathrm{s}^{-1}$ or LU) of the central emission feature together with its model fit parameters and their associated $\chi^{2}$ fitting errors and a measure of the the detection significance with respect to the level of noise in the data (both with and without the presence of the emission feature of interest). The fit procedure also takes into account the limited resolution of the
SPEAR data, such that all interstellar emission lines with wavelengths close to the line of interest are also fit simultaneously. In the case of the CIV emission lines at $1548 \AA$ and $1551 \AA$ the observed profile was fit simultaneously as a doublet feature and also taking into account the presence of the nearby SiII* emission line. Because of the close proximity of the strong Lyman beta airglow feature, the O VI lines at $1032 \AA$ and $1038 \AA$ were fit with empirical emission line profiles (constructed from the Lyman beta line) instead of a pure Gaussian profile. The underlying continuum background for these lines was determined empirically by adding many spectra where O VI was not detected. The resulting model was thus the sum of Lyman Beta, the two O VI features, the C II features (1036.34 and 1037.02 $\AA$ ), and the continuum background. The free parameters of the fit were the intensities of the various emission lines, the intensity of the background, and the wavelength centroid of the Lyman Beta line. The wavelengths of the other features were kept as constant offsets relative to the Lyman Beta line.

In Figs. 3a-c we show typical examples to the quality of the fits to some of the line spectra for several of the (large) skypixels. The final result of this line-fitting process are 2D maps 

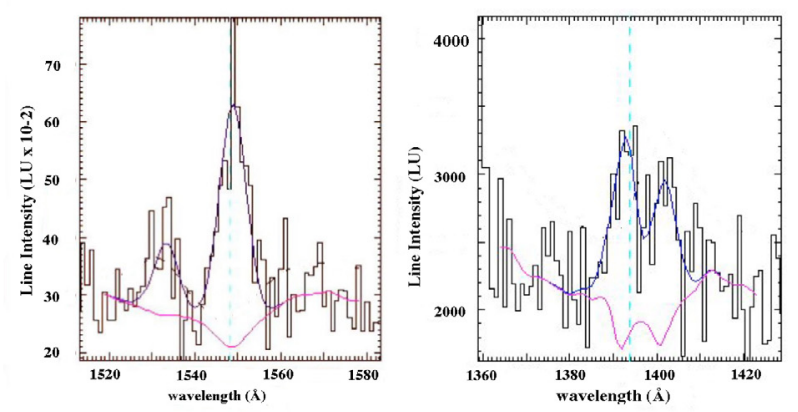

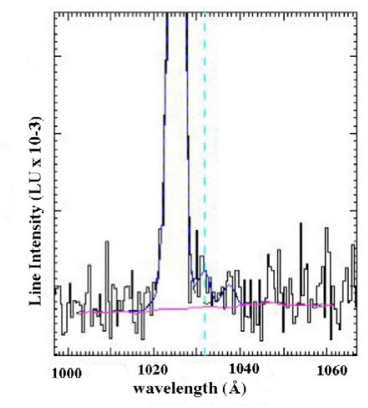

Fig. 3. Typical emission line fits to the CIV doublet at $1550 \AA$, the SiIV doublet at $1394 \AA$ and the OVI $1032 \AA$ doublet. The lower full line represents the best-estimate of the underlying dust-scattered stellar continuum.

Table 1. SPEAR measured FUV emission line intensities (in LU) averaged over $8^{\circ} \times 8^{\circ}$ bins at sky positions A-P.

\begin{tabular}{|c|c|c|c|c|c|c|c|c|c|}
\hline$\overline{\text { SkyPixel }}$ & $\bar{l}$ & $\overline{\bar{b}}$ & $\begin{array}{c}I(\mathrm{OVI}) \\
\text { (scaled doublet) } \\
(\mathrm{LU})\end{array}$ & $\begin{array}{c}\text { I(CIV) } \\
\text { (doublet) } \\
(\text { LU })\end{array}$ & $\begin{array}{c}I(\text { SiIV }) \\
\text { (scaled doublet) } \\
(\mathrm{LU})\end{array}$ & $\begin{array}{c}I(\mathrm{SiII} *) \\
(\mathrm{LU})\end{array}$ & $\begin{array}{c}\text { (AlII) } \\
(\mathrm{LU})\end{array}$ & $\overline{I(\mathrm{OVI}) /(\mathrm{CIV})}$ & $\overline{I I(\mathrm{CIV}) / I(\mathrm{SiIV})}$ \\
\hline $\mathrm{A}$ & $301.5^{\circ}$ & $+62.8^{\circ}$ & $21160 \pm 7500$ & $7840 \pm 660$ & $3530 \pm 610$ & $2995 \pm 655$ & $6035 \pm 1780$ & $2.7 \pm 0.98$ & $2.2 \pm 0.55$ \\
\hline B & $312.8^{\circ}$ & $+71.5^{\circ}$ & $<6300$ & $7770 \pm 580$ & $4290 \pm 2185$ & $1830 \pm 690$ & $4230 \pm 1080$ & $<0.8$ & $1.8 \pm 1.0$ \\
\hline $\mathrm{C}$ & $341.6^{\circ}$ & $+78.8^{\circ}$ & $16420 \pm 8000$ & $7850 \pm 310$ & $2610 \pm 300$ & $2580 \pm 335$ & $6335 \pm 2090$ & $2.1 \pm 1.1$ & $3.0 \pm 0.48$ \\
\hline $\mathrm{D}$ & $303.8^{\circ}$ & $+78.5^{\circ}$ & $<6200$ & $6890 \pm 405$ & $2215 \pm 420$ & $2355 \pm 455$ & $3400 \pm 1130$ & $<0.9$ & $3.1 \pm 0.71$ \\
\hline $\mathrm{E}$ & $292.0^{\circ}$ & $+69.1^{\circ}$ & $10320 \pm 5500$ & $6350 \pm 450$ & $2490 \pm 765$ & $1925 \pm 445$ & $3090 \pm 1515$ & $1.6 \pm 0.96$ & $2.6 \pm 0.98$ \\
\hline $\mathrm{F}$ & $287.5^{\circ}$ & $+59.7^{\circ}$ & $<6300$ & $8105 \pm 530$ & $3330 \pm 485$ & $2200 \pm 430$ & $6285 \pm 1200$ & $<0.8$ & $2.4 \pm 0.50$ \\
\hline $\mathrm{G}$ & $276.3^{\circ}$ & $+55.4^{\circ}$ & $15830 \pm 6500$ & $6515 \pm 455$ & $2365 \pm 630$ & $1475 \pm 410$ & $4930 \pm 1290$ & $2.4 \pm 1.1$ & $2.8 \pm 0.92$ \\
\hline $\mathrm{H}$ & $276.3^{\circ}$ & $+64.9^{\circ}$ & $9350 \pm 4200$ & $4695 \pm 385$ & $2140 \pm 385$ & $1860 \pm 405$ & $4370 \pm 920$ & $2.0 \pm 1.0$ & $2.2 \pm 0.57$ \\
\hline I & $276.3^{\circ}$ & $+74.7^{\circ}$ & $<4000$ & $3670 \pm 365$ & $1565 \pm 370$ & $1320 \pm 320$ & $3860 \pm 1525$ & $<1.1$ & $2.3 \pm 0.76$ \\
\hline J & $276.2^{\circ}$ & $+84.8^{\circ}$ & $6765 \pm 3900$ & $5775 \pm 225$ & $1830 \pm 220$ & $1910 \pm 315$ & $4260 \pm 1470$ & $1.2 \pm 0.7$ & $3.2 \pm 0.50$ \\
\hline $\mathrm{K}$ & $248.7^{\circ}$ & $+78.5^{\circ}$ & $10000 \pm 5000$ & $4390 \pm 345$ & $2140 \pm 700$ & $1435 \pm 295$ & $4700 \pm 1310$ & $2.3 \pm 1.3$ & $2.1 \pm 0.84$ \\
\hline $\mathrm{L}$ & $260.6^{\circ}$ & $+69.1^{\circ}$ & $<5050$ & $3175 \pm 520$ & $1975 \pm 510$ & $2615 \pm 470$ & $3595 \pm 920$ & $<1.6$ & $1.6 \pm 0.67$ \\
\hline M & $265.1^{\circ}$ & $+59.7^{\circ}$ & $<6000$ & $4715 \pm 645$ & $2855 \pm 840$ & $1540 \pm 470$ & $6020 \pm 1525$ & $<1.3$ & $1.7 \pm 0.73$ \\
\hline $\mathrm{N}$ & $251.1^{\circ}$ & $+62.8^{\circ}$ & $<6200$ & $2035 \pm 730$ & $<1200$ & $<600$ & $<2500$ & $<3.0$ & $>1.7$ \\
\hline $\mathrm{O}$ & $239.8^{\circ}$ & $+71.5^{\circ}$ & $<6580$ & $4455 \pm 570$ & $<1400$ & $2300 \pm 550$ & $3275 \pm 840$ & $<1.5$ & $>3.1$ \\
\hline $\mathrm{P}$ & $211.1^{\circ}$ & $+78.7^{\circ}$ & $<7500$ & $4150 \pm 600$ & $<1300$ & $1050 \pm 505$ & $3315 \pm 825$ & $<1.8$ & $>3.2$ \\
\hline N.E.P.** & $96.0^{\circ}$ & $+29^{\circ}$ & $5725 \pm 570$ & $5820 \pm 280$ & $1430 \pm 120$ & $2430 \pm 200$ & $\mathrm{~N} / \mathrm{A}$ & $0.98 \pm 0.15$ & $4.1 \pm 0.53$ \\
\hline
\end{tabular}

** Oservations of the North Ecliptic Pole (NEP) region by Korpela et al. (2006).

(in galactic co-ordinates) of the emission line intensities (in LU) contained within spatial bins of 64 sq. deg. on the sky. In Figs. 4 and 5 we show the relevant line-maps of the CIV (1550 $\AA$ doublet), SiIV (1394 ̊), OVI (1032 ̊ doublet), SiII* (1533 $\AA$ ) and AlII (1671 $\AA$ ) emission (in LU) as a function of position on the sky. In these plots we have labeled 16 of the $8^{\circ} \times 8^{\circ}$ sky-pixels with letters from A to $\mathrm{P}$, with their associated area averaged emission line intensities being listed in Table 1 together with their associated measurement errors. We note the quite different contributions to the overlying emission line intensities from the underlying model stellar continua for each line. In general we report line detections and fits with a confidence level of $>3 \sigma$ significance, but in order to present results for the weakly detected OVI line we have reduced the confidence level for this line to detections with $>2 \sigma$ significance. We also note that the interstellar SiIV line at $1394 \AA$ is blended with that of both SiIV (1402 ̊) and OIV] (1400 ̊) emission, which Korpela et al. (2006) have estimated to possess a comparable emission intensity. Therefore, since we do not know the exact contribution from OIV to the SiIV (1402 $\AA$ ) line, we shall assume a 2:1 ratio for the SiIV doublet components (based on their oscillator strengths), and thus we report 1.5 times the measured value of the SiIV (1394 A) line as being representative of the total doublet value for that ion species. Similarly we have scaled the measured intensity of the (stronger) OVI (1032 $\AA$ ) line to report a value for the OVI doublet, based on the oscillator strength of the weaker OVI (1038 $\AA$ ) line. The intensity value for CIV reported in Table 1 is for the fitted line-doublet, whereas the emission levels reported for both SiII* and AlII are for the single lines only. Finally, in the last row of Table 1 we show (for comparison purposes) the equivalent emission line data for the North Ecliptic Pole region as measured with SPEAR (Korpela et al. 2006).

It should be noted that we have made no attempt to correct the line intensity values listed in Table 1 for extinction by interstellar dust. Figure $4 d$ shows that the general level of extinction is low in most sky-pixels (i.e. $E(B-V)<0.05 \mathrm{mag}$ ). However, it should be noted that even this (small) amount of dust can reduce the flux at the OVI $1032 \AA$ line by a factor of 2 . Since the precise location of the source of UV line emission with respect to dust absorption is not well-known from our $8^{\circ} \times 8^{\circ}$ maps, we can only refer the reader to the extinction values shown in Fig. $4 \mathrm{~d}$ for an estimation of the possible levels of emission line attenuation for each sky-pixel.

\section{Discussion}

\subsection{The CIV emission map}

Figure 4a clearly shows that the greatest line-emission intensity from this ion is found along the left-hand edge of the region under investigation (i.e. the strip of sky with galactic longitude $l>290^{\circ}$ ). The emission intensity along this edge (within skypixels $\mathrm{A}$ to $\mathrm{F}$ ) is typically $>6500 \mathrm{LU}$, which is to be compared with that of $<4500 \mathrm{LU}$ for the remainder of the region (skypixels $\mathrm{K}$ to $\mathrm{P}$ ). These emission levels are numerically consistent with those observed by Martin \& Bowyer (1990) who found 

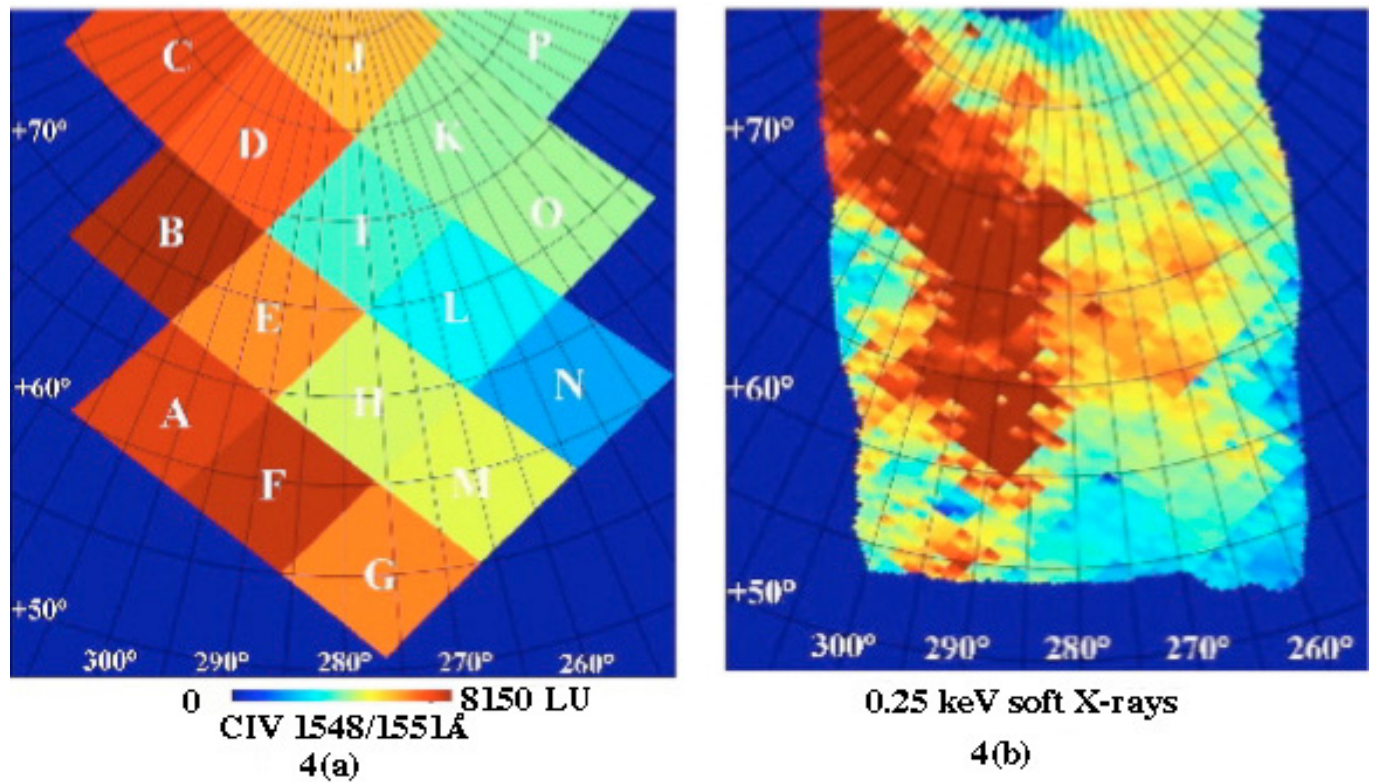

4(b)

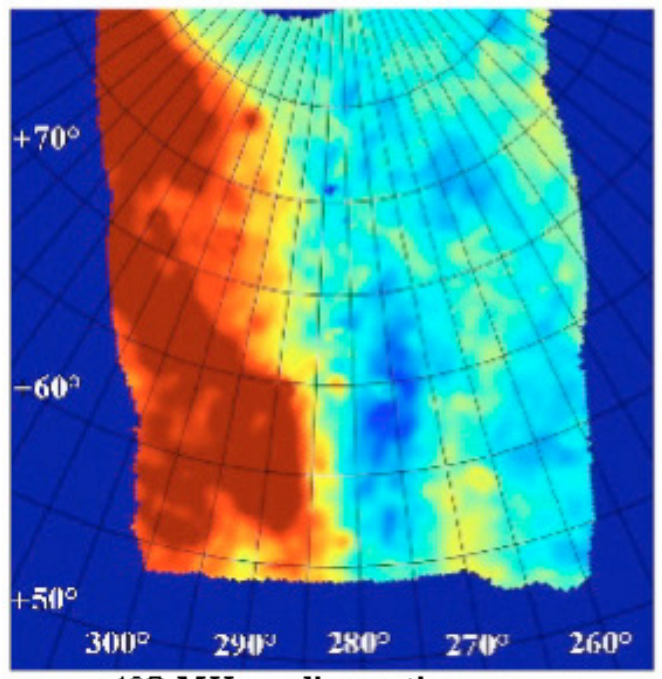

$408 \mathrm{MHz}$ radio continuum 4(c)

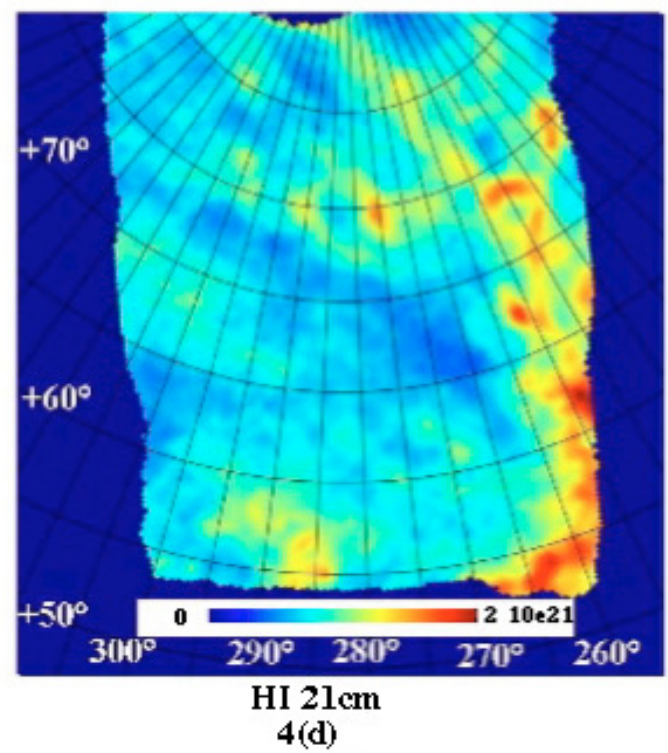

Fig. 4. a) SPEAR CIV emission line intensity map for the North Galactic Pole region. The highest levels of emission (shown in dark red) are found in sky-pixels labeled A, B, C, D and F, which are also spatially coincident with the high levels of $0.25 \mathrm{keV}$ soft X-ray emission from the edge of North Polar Spur feature shown in b). Maps of $408 \mathrm{MHz}$ radio continuum and $\mathrm{HI} 21 \mathrm{~cm}$ radio emisison from the same region of the sky are also shown in c) and d). All emission intensity scales are linear.

CIV line (doublet) intensities in the range 2200-5700 LU for similarly high latitude halo gas.

In Fig. $4 \mathrm{~b}$ we show the same region of the sky recorded at $0.25 \mathrm{keV}$ soft X-ray wavelengths with the ROSAT all-sky survey (Snowden et al. 1997), and similarly in Fig. 4c we show the $408 \mathrm{MHz}$ radio continuum emission (Haslam et al. 1982) and in Fig. 4d the corresponding $21 \mathrm{~cm} \mathrm{HI}$ radio emission (Hartmann \& Butler 1997). We note that this latter map is very similar to that of the distribution of dust IR emission as recorded by the COBE and IRAS satellites (Schlegel et al. 1998). These three maps have been constructed using the HEALpix routines of Gorski et al. (2005), typically with spatial resolutions $<10$ arcmin. A comparison of these maps with that of the CIV line-intensity distribution in Fig. 4a clearly reveals three main aspects: (i) there is a high spatial correlation between regions of high CIV line-emission intensity and high intensity values of $408 \mathrm{MHz}$ radio contin- uum emission; (ii) the region of high CIV line-emission intensity lies adjacent to, but not completely co-aligned with, that of the highest levels of soft X-ray emission; and (iii) there is an anticorrelation between regions of high gas and dust emission and those of high CIV, high $408 \mathrm{MHz}$ and high soft X-ray emission intensity. This latter result is not unexpected, since even small amounts of neutral gas and interstellar dust can easily shadow or totally absorb soft X-ray photon and $408 \mathrm{MHz}$ emission from fast moving electrons, in addition to efficiently scattering UV photons.

Willingdale et al. (2003) have analyzed the soft X-ray background signal in this direction and found that the NPS and halo emission components lie behind at least 50 per cent of the line-of-sight cold gas. The cold (HI) gas clouds are thought to be located at distances over the range 120-200 pc in this general direction, as determined by both photometry 
(Haikala et al. 1995) and $\mathrm{NaI}$ absorption studies towards nearby early-type stars (Lallement et al. 2003). The largest concentration of HI (and dust) clouds is located at galactic longitudes $<265^{\circ}$, with an estimated neutral interstellar HI column density of $>10^{20} \mathrm{~cm}^{-2}$, whereas the remainder of the area is composed of numerous filamentary clouds with a typical HI column density $<6 \times 10^{19} \mathrm{~cm}^{-2}$ (based on NaI observations towards the star HD 109860 of distance 200 pc, Lallement et al. 2003). We also note that the reddening towards most of this region has a low value of $E(B-V)<0.05$. Since the region of low-level CIV emission $\left(l=265-275^{\circ}\right.$, sky-pixels $\mathrm{K}$ to $\left.\mathrm{P}\right)$ is spatially coincident with that of significantly reduced soft X-ray emission, it is reasonable to assume (like Willingdale et al. 2003) that a significant proportion of this diffuse ionized gas emission lies behind the foreground neutral HI gas clouds at distances $>200 \mathrm{pc}$ in the overlying halo. Such gas can be identified with that commonly traced by absorption studies of the CIV and SiIV ions detected routinely towards halo stars with distances of $z<4 \mathrm{kpc}$ (Savage et al. 1997).

The NPS feature has been conjectured to be a nearby supernova remnant (Berkhuijsen et al. 1971), or an ionized outflow from stellar winds of the underlying Sco-Cen OB association (Egger \& Aschenbach 1995). We note that at high galactic latitudes the NPS, as seen both in radio and X-ray emission, appears as a limb-brightened shell, as may be expected from the perspective of an expanding supernova remnant. Our presently discovered enhancement of CIV line-emission is seen in regions facing towards the (lower galactic latitude) NPS, for galactic longitudes $>285^{\circ}$. This picture would seem to support the notion that the region of the highest intensity $408 \mathrm{MHz}$ radio continuum emission (which is caused by synchrotron processes) is associated with shocks that delineate the outer shell of the NPS and are also contributing to the observed high levels of CIV emission. The region with galactic longitudes $<285^{\circ}$ lies beyond the giant NPS feature and is probably representative of the general diffuse halo gas with a lower associated CIV emission line intensity of $\sim 4000$ LU.

\subsection{The SilV emission map}

Figure 5a shows the spatial distribution of SiIV (1394 A) line emission, with the highest emission levels occurring in skypixels A, B and F with intensities $>3300$ LU. Apart from skypixel $\mathrm{M}$, the remainder of the region is characterized by lowerlevel SiIV emission typically with a value $<2600 \mathrm{LU}$. We note that although sky-pixels A, B and F are also associated with similarly high levels of CIV line emission, the spatial distribution of SiIV emission is not as well-correlated with the highest levels of both $0.25 \mathrm{keV}$ soft X-ray and $408 \mathrm{MHz}$ radio emission that trace the outer edge of the NPS (shell) feature. We note that SiIV emission is normally associated with warm and ionized gas (often the sites of small-scale mixing layers or conductive thermal fronts) at a temperature of $\sim 60000 \mathrm{~K}$, in contrast with the far higher temperature $\left(T \sim 10^{6} \mathrm{~K}\right)$ and more wide-spread soft $\mathrm{X}$-ray emitting regions. The very lowest levels of SiIV emission were recorded as upper limits (<1400 LU) in the sky-pixels with galactic longitudes $<270^{\circ}$. We confirm the notion (forwarded in the previous section) that such regions are unconnected with the NPS shell feature and presumably associated with general halo gas emission. This is in accord with the observations of the North Ecliptic Pole (NEP) region with SPEAR by Korpela et al. (2006), who measured an emission level of $1430 \mathrm{LU}$ for the SiIV doublet.

\subsection{The OVI emission map}

Figure 5b shows the spatial distribution of the OVI (1032 $\AA$ ) line emission. Due to both the relative weakness of emission from this line and the associated difficulty in its detection due to the proximity to the strong geocoronal Lyman Beta line, firm detections were made in only 7 of the 16 sky-pixels. The highest observed emission levels were found in sky-pixels A and C, which can be associated with positions of the highest CIV, soft X-ray and $408 \mathrm{MHz}$ emission within the edge of the NPS feature. Our detection lower limit (for the OVI doublet) is 6200 LU, which is marginally above the level of 5725 LU measured in extended SPEAR observations of the NEP region by Korpela et al. (2006). We note that regions from which we have been unable to measure detectable levels of OVI emission (e.g. sky-pixels B, $\mathrm{F}, \mathrm{M}, \mathrm{N}$ and $\mathrm{O}$ ) can apparently be associated with the presence of foreground HI gas clouds shown in Fig. 4d. Such clouds may account for the clumpy nature of the observed distribution of the OVI ion.

Emission from the OVI line is generally associated with highly ionized gas in collisional equilibrium with a temperature of $\sim 300000 \mathrm{~K}$. Such gas cools rapidly and thus this ion traces interstellar regions in transition either through cooling or at the interfaces between warm and hotter gas. Such sites are prime regions for the formation of both CIV and SiIV ions at a temperature of $\sim 10^{5} \mathrm{~K}$. In a recent survey of diffuse OVI emission by Dixon et al. (2006) using the FUSE satellite, they observed 5010 LU of OVI (1032 $\AA$ ) emission from a region of area $30 \operatorname{arcsec} \times 30 \operatorname{arcsec}\left(l=284^{\circ}, b=+74.5^{\circ}\right)$, which is comparable to the average level of $\sim 6800 \mathrm{LU}$ (for the OVI $1032 \AA$ line), scaled from observations of the OVI doublet recorded in the $8^{\circ} \times 8^{\circ}$ sky-pixels E \& $\mathrm{K}$ by SPEAR. For comparison purposes, the associated level of soft X-ray flux at this position on the sky is $4520 \times 10^{-6}$ counts s arcmin $^{-2}$ (Snowden et al. 1997).

\subsection{The Sill* emission map}

Figure $5 \mathrm{c}$ shows the spatial distribution of emission from the SiII* (1533 Å) line, which primarily exists over an ionization potential range of 8.2 to $16.3 \mathrm{eV}$ and thus traces ionized and partially ionized/neutral gas regions. Under collisional ionization conditions its maximum emission occurs at a temperature of $\sim 12500 \mathrm{~K}$. The present non-detection of the nearby SiII (1526 $\AA$ ) line (see Fig. 2) is attributed to opacity effects (Korpela et al. 2006; Shinn et al. 2006). Although the highest emission levels of SiII* $(\sim 2500 \mathrm{LU})$ are to be found in sky-pixels A to F (which are spatially coincident with significant soft X-ray and CIV emission from the NPS feature), similarly high levels of emission can also be found in sky-pixels $\mathrm{L}$ and $\mathrm{O}$ which are thought to be associated with the diffuse gas of the halo. We note that SiII* is the most abundant ionization state of silicon in the warm and neutral (weakly ionized) regions of the ISM. This is in contrast to regions of high CIV emission intensity which generally trace either collisionally ionized or highly photo-ionized interstellar regions. There is some similarity between both emission maps for positions near to the edge of the NPS (sky-pixels A to $\mathrm{F}$ ), whereas pixels $\mathrm{L}$ and $\mathrm{O}$ are clearly anomalous in their observed high level of SiII* emission. Finally, we note that the level of SiII* from the general halo gas is $\sim 2500 \mathrm{LU}$, which is in agreement with a level of $2430 \mathrm{LU}$ recorded by Korpela et al. (2006) towards the NEP region. 

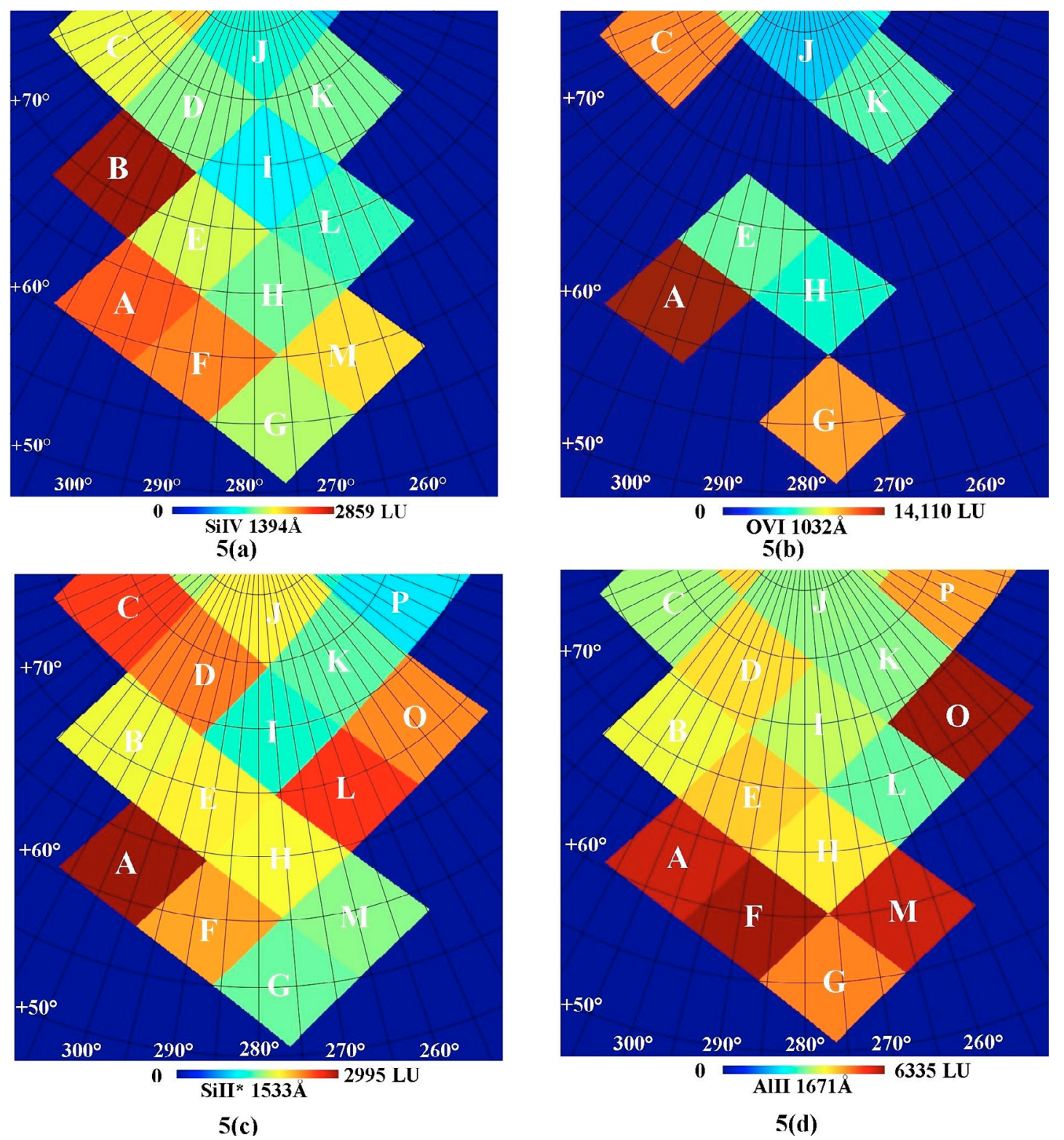

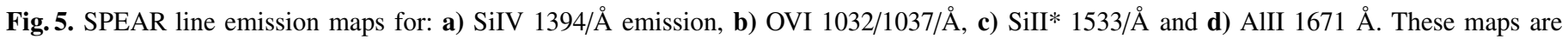
directly comparable with those shown in Fig. 4 for the same region of the sky.

\subsection{The All emission map}

Figure 5d shows the spatial distribution of the AlII (1671 $\AA$ ) emission. Following the work of Korpela et al. (2006), we see no evidence for any measurable emission from the nearby OIII $(1665 \AA)$ emission line in our spectral data. The prior detection of the OIII line by the lower spectral resolution $(\sim 20 \AA)$ observations of Martin \& Bowyer (1990) was probably due to a misidentification of the detection of emission from the adjacent AlII line. The AlII ion exists over the ionization potential range of 6.0 to $18.8 \mathrm{eV}$ and under collisional ionization conditions has its maximum emission at a temperature of $\sim 20000 \mathrm{~K}$. Its relative strength throughout the presently mapped region suggests that warm-(partially)-neutral gas is quite pervasive in the halo, thus supporting the notion that the halo is not isothermal in nature. Although Fig. 5d shows appreciable levels of AlII line emission over the whole mapped area, the very highest intensities occur in sky-pixels A, C, F and M. These sky-positions correlate well with those of the denser (and presumably colder) $21 \mathrm{~cm}$ HI clouds shown in Fig. 4d.
Finally we note that Korpela et al. (2006) observed an AlII emission intensity level of 5610 LU towards the NEP region, which is $\sim 20 \%$ higher than the average level detected across the whole of the presently mapped region.

\section{FUV line ratio analysis}

\subsection{The high ions}

In Table 1 we list two important emission line intensity ratios for the sky-pixels sampled with SPEAR; that of $I(\mathrm{OVI}) / I(\mathrm{CIV})$ and $I(\mathrm{CIV}) / I(\mathrm{SiIV})$. Such ratios are indicative of the presence of ionized gas with a temperature $>60000 \mathrm{~K}$. Although much work has been presented on the equivalent ratios from the corresponding interstellar absorption lines of OVI, CIV and SiIV (Zsargo et al. 2003; Sembach et al. 2003; Indebetouw \& Shull 2004b), very little work currently exists on measurement of the line ratios recorded in emission from the general diffuse IS gas. However, several theoretical models currently exist that do make predictions for the expected high ion emission measures based on 
various assumptions generally concerning the relative importance of either collisional or photo-ionzation processes (CLOUDY: Ferland 2003, CHIANTI: Young et al. 2003). For a comprehensive list of the major production mechanisms for these high ions we refer the reader to the work of Indebetouw \& Shull (2004a). At high galactic latitudes, the most presently favored production mechanism for halo high ions is that of the galactic fountain (Shapiro \& Field 1993), in which hot gas is liberated into the overlying halo from expanding supernova remnants and/or stellar bubbles that originate in the galactic disk. However, the presence of the NPS feature (which is thought to be delineated by a highly ionized shocked superbubble or SNR shell), should perhaps argue for a different production mechanism for the high ions located at galactic longitudes $>285^{\circ}$. Bearing this in mind, we might expect the high ion ratios to be different in gas associated with the (shocked) North Polar Spur region (sky-pixels A-F) compared with that of the general halo, thus providing us with a diagnostic tool that can be used to infer differences in the various emission production mechanisms.

Firstly we note that the $I(\mathrm{OVI}) / I(\mathrm{CIV})$ ratio (when measured) gives values in the relatively restricted range of 1.2 to 2.7, with an typical error of $\sim \pm 1.0$. For sky-positions in which the OVI line was not detected with confidence (but was detected in CIV), an average value of $I(\mathrm{OVI}) / I(\mathrm{CIV})<1.4$ can be assigned to these regions, which is similar to that of $I(\mathrm{OVI}) / I(\mathrm{CIV})=0.98$ measured for diffuse halo gas near the North Ecliptic Pole by Korpela et al. (2006). Values of the $I(\mathrm{OVI}) / I(\mathrm{CIV})$ ratio in the 0.9 to 3.9 range have been reported for emission from highly ionized gas associated with fast moving shocks in SNRs (Raymond et al. 1997; Danforth et al. 2001; Sankrit et al. 2003). Thus, perhaps surprisingly, the range of values of the $I(\mathrm{OVI}) / I(\mathrm{CIV})$ ratio presently measured by us towards the entire region is very similar to that determined for high velocity gas associated with SNR shocks.

Our range of measured $I(\mathrm{OVI}) / I(\mathrm{CIV})$ ratio values is consistent with that predicted by the highly ionized halo gas model forwarded by Shull \& Slavin (1994), which incorporates contributions from both turbulent mixing layers (Slavin et al. 1993) and isobarically cooling supernova remnants. Based on an assumed absorption ratio of $N(\mathrm{OVI}) / N(\mathrm{CIV})=3$ for a typical halo sightline, they predict an emission ratio of $I(\mathrm{OVI}) / I(\mathrm{CIV})=1.2 \pm 0.2$ for emitting halo gas with a temperature of $\log T=5.3 \pm$ $0.3 \mathrm{~K}$, an electron density of $0.012 \mathrm{~cm}^{-3}$ and a gas pressure of $P / k=2400 \mathrm{~cm}^{-3}$. The actual values of high ion absorption measured towards the nearest halo star to our presently mapped region of the sky (HD 100340: $\left.l=259^{\circ}, b=+61^{\circ}\right)$, is $N(\mathrm{OVI}) / N(\mathrm{CIV})=2.8$ (Sembach et al. 1997; Zsargo et al. 2003), which is very similar to that assumed in the Shull \& Slavin calculations. We note that our presently measured values of the $I(\mathrm{OVI}) / I(\mathrm{CIV})$ ratio are inconsistent with the galactic fountain model predictions of Edgar \& Chevalier (1986) and Houck \& Bregman (1990) which respectively predict ratios an order of magnitude higher and lower than those presently measured. However, we shall not pursue specific model predictions in further detail since many of the input parameters of other models (such as that of Shelton 1998), can be suitably altered to produce results close to our presently measured values. We therefore leave a more detailed interpretation of our present SPEAR observations to more qualified theorists.

In order to make a (very) approximate estimate of the the thermal pressure $(P / k)$ of hot $(T=300000 \mathrm{~K})$ collisionally ionized gas in the halo (of which there are only a few other measurements), we use the FUV absorption measurements of Savage et al. (2003) who observed $\log N(\mathrm{OVI})=14.31 \mathrm{~cm}^{-2}$ towards
NGC 4649 (in sky-pixel "C") and $\log N(\mathrm{OVI})=14.73 \mathrm{~cm}^{-2}$ towards 3C 273 (in sky-pixel "J"). If we make the assumption that the results from the SPEAR OVI emission data (averaged over 64 sq. deg on the sky) can be meaningfully compared with the absorption data (gained from pencil beam measurements with FUSE), then we may derive electron density values, $\mathrm{n}_{e}$, from the respective $I(\mathrm{OVI}) / N(\mathrm{OVI})$ ratios scaled to those given in Shelton et al. (2001). From these values of $n_{\mathrm{e}}$ we derive estimates of $P / k=2.1 \times 10^{4} \mathrm{~cm}^{-3}$ for hot halo gas in sky-pixel " $\mathrm{C}$ " and $P / k=3.3 \times 10^{3} \mathrm{~cm}^{-3}$ in sky-pixel "J". We thus find a difference in hot gas pressure of $\sim$ a factor 7 between the two regions sampled. Recent observations of OVI in the galactic halo by Shelton et al. (2007) give gas pressures closer to those calculated for skypixel "J".

Finally, we note that the relatively restricted range of the I(OVI)/I(CIV) ratio observed by both our SPEAR observations and observations of SNR shocked gas would seem to argue in favor of a common production mechanism for both ions. Whenever a high level of OVI emission is observed it is always accompanied by (higher than average) CIV emission with an intensity $>4300$ LU. This is probably best explained under the assumption that OVI halo emission is somewhat clumpy in nature, consistent with its production at interfaces between warm $\left(T=10^{3}-10^{4} \mathrm{~K}\right)$ and hotter $\left(T=10^{6} \mathrm{~K}\right)$ soft X-ray emitting gas. The associated CIV emission at such interfaces is thought to occur in the intermediate temperature $\left(T=10^{5} \mathrm{~K}\right)$ gas, which seems always present whenever OVI is strongly detected. An alternate explanation for our results is that CIV emission may be ubiquitous throughout the halo with an associated line intensity (of $\sim 4000 \mathrm{LU}$ ) that is fairly constant, and our present observations merely reflect a superposition of OVI emission that originates at the interfaces of random clumps of emitting gas in the halo.

The values of the $I(\mathrm{CIV}) / I(\mathrm{SiIV})$ ratio listed in Table 1 span the range 1.6 to 3.2 , with a typical measurement error of $\sim \pm 0.8$. Korpela et al. (2006) report a value of $I(\mathrm{CIV}) / I(\mathrm{SiIV})=4.1$ for the North Ecliptic Pole, which is an interstellar region thought to sample diffuse, warm and ionized halo gas Although much work exists on the measurement of the $N(\mathrm{CIV}) / N(\mathrm{SiIV})$ ratio derived from halo gas absorption studies (Sembach \& Savage 1992), the emission ratio of $I(\mathrm{CIV}) / I(\mathrm{SiIV})$ (like that of the $I(\mathrm{OVI}) / I(\mathrm{CIV})$ ratio)has only been observed for gas associated with strong, fast moving shocks associated with SNRs or expanding superbubbles (Raymond et al. 1997; Danforth et al. 2001; Sankrit et al. 2003). In such cases, values of the $I(\mathrm{CIV}) / I$ (SiIV) ratio in the range 1.9 to 3.9 are typically observed. Thus, again our present observations of a high ion line emission ratio do not seem to provide us with a sensitive diagnostic that can differentiate between emission from shocked or the general diffuse and ionized halo gas.

Since the SiIV ion is normally associated with lower temperature interstellar regions, as opposed to the higher temperature and higher ionization lines of NV, CIV and OVI, its contribution to the total emission from $>10^{5} \mathrm{~K}$ gas has often been omitted from models that make line intensity predictions, apart from those regions associated with SNR shocks. Further work on theoretical predictions are thus clearly required for the more general case of diffuse and ionized halo gas.

\subsection{The lower ions}

Both the SiII* and AlII ions trace mainly diffuse and warm(partially)neutral interstellar regions with a gas temperature $<20000 \mathrm{~K}$. This type of gas seems quite pervasive in the halo 
and the distribution of AlII seems well-correlated with that of $21 \mathrm{~cm} \mathrm{HI}$ emitting gas. We derive ratio values of $I\left(\mathrm{SiII}^{*}\right) / I(\mathrm{AlII})$ that span the fairly restricted range of $0.25-0.7$ over the whole region, which is consistent with the value of $0.43 \pm 0.8$ derived by Korpela et al. (2006) for the North Ecliptic Pole region. In contrast, Kregenow et al. (2006) derive a significantly higher ratio of 1.1 for gas associated with an evolved (hot) thermal interface in the Eridanus Loop superbubble.

We also note from Table 1 that the pixels with the highest values of CIV emission (A-G) possess values of $I(\mathrm{CIV}) / I(\mathrm{AlII})$ in the range 1.3-2.1, whereas sky-pixels with the lowest emission levels of CIV (K-P) possess ratio values only in the $0.8-1.4$ range. This could be somewhat significant, since the former set of pixels can be firmly associated with the hot and highly ionized shell of the NPS feature, whereas the latter pixels are thought to sample the general diffuse halo gas that is spatially distant from the local influence of shocks and/or strong sources of photo-ionization.

\section{Conclusion}

We have presented far ultraviolet (FUV: 1350-1750 A) spectral imaging observations recorded with the SPEAR satellite of

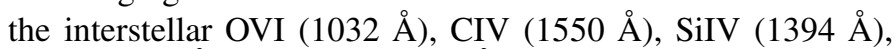
SiII* $(1533 \AA)$ and AlII (1671 $\AA$ ) emission lines originating in a $60^{\circ} \times 30^{\circ}$ rectangular region lying close to the North Galactic Pole. These data represent the first large area, moderate spatial resolution maps of the distribution of UV spectralline emission originating the both the highly ionized medium (HIM) and the warm ionized medium (WIM) recorded at high galactic latitudes. Our maps of the OVI, CIV, SiIV and SiII* line emission show the highest intensity levels being spatially coincident with similarly high levels of soft X-ray emission originating in the edge of the Northern Polar Spur interstellar feature. However, the distribution of the low ionization AlII emission does not show a similar spatial correlation, which suggests that warm-neutral and/or partially ionized gas with a temperature $<20000 \mathrm{~K}$ may be quite pervasive at high galactic latitudes.

The observed emission line intensity ratios for both high and low ions are discussed, and the variation in these ratios as a function of position on the sky is contrasted with the predictions from current theories concerning the physical state of the galactic halo. Our data indicates that these various line ratios alone do not provide us with a clear diagnostic tool to distinguish between the various physical production mechanisms for both high and low ion states. However, a comparison between the spatial distribution and morphology of UV, radio and soft X-ray emission from this region does allow us to draw meaningful conclusions concerning the possible production mechanisms for highly ionized gas at high galactic latitudes. Our present data favors the hybrid model of Shull \& Slavin (1994), which incorporates contributions from both turbulent mixing layers and isobarically cooling supernova remnants.

Our observed spatial distribution of highly ionized gas near the North Galactic Pole region can be best explained under the assumption that OVI halo emission is somewhat clumpy in nature, consistent with its production at interfaces between warm $\left(T=10^{3}-10^{4} \mathrm{~K}\right)$ and hotter $\left(T=10^{6} \mathrm{~K}\right)$ soft X-ray emitting gas. The associated CIV emission at such interfaces occurs in the intermediate temperature $\left(T=10^{5} \mathrm{~K}\right)$ gas, which seems always present (at a higher than average level) whenever OVI is strongly detected. An alternate explanation for these observations is that CIV emission is ubiquitous throughout the halo with an associated fairly constant level of emission line intensity (of $\sim 4000$ LU), and therefore our observations may generally reflect the superposition of spatially separate OVI emission which originates at the cloud interfaces of random clumps of high latitude gas.

Finally we note that although these observations represent a unique data set, our present conclusions are highly dependent on the limited sensitivity and moderate spectral resolution of the SPEAR instrument. It is clear that more sensitive and higher spectral resolution emission observations of the FUV sky are required to answer many of the outstanding problems associated with the presence and physical state of highly ionized emitting gas in our Galaxy.

Acknowledgements. SPEAR/FIMS is joint space project of KASI \& KAIST (Korea) and U.C. Berkeley (USA), funded by the Korea MOST and NASA grant NAG5-5355. We acknowledge the dedicated team of engineers, technicians, and administrative staff from SSL, SaTReC and KASI who made this mission possible. This publication makes use of data products from the SIMBAD database, operated at CDS, Strasbourg, France.

\section{References}

Berkhuijsen, E., Haslam, C., \& Salter, C. 1971, A\&A, 14, 252

Black, J. H., \& van Dishoeck, E. 1987, ApJ, 203, 132

Bowyer, S. 1991, ARA\&A, 29, 59

Burton, W. B., \& Hartmann, D. 1994, Ap\&SS, 217, 189

Cen, R., \& Ostriker, J. 1999, ApJ, 514, 1

Danforth, C., Blair, W., \& Raymond, J. 2001, AJ, 122, 938

Dixon, W.V., Sankrit, R., \& Otte, B. 2006, ApJ, 647, 328

Edelstein, J., Min, K. W., Han, W., et al. 2006a, ApJ, 644, L153

Edelstein, J., Korpela, E., Min, K., et al. 2006b, ApJ, 644, L159

Edgar, R., \& Chevalier, R. 1986, ApJ, 310, L27

Egger, R., \& Aschenbach, B. 1995, A\&A, 294, L25

Ferland, G. J. 2003, ARA\&A, 41, 517

Finkbeiner, D., Davis, M., \& Schlegel, D. 1999, ApJ, 524, 867

Gorski, K., Hivon, E., Banday, A., et al. 2005, ApJ, 622, 759

Haffner, L., Reynolds, R., Tufte, S., et al. 2003, ApJS, 149, 405

Haikala, L., Mattila, K., Bowyer, S., et al., 1995, ApJ, 443, L33

Hartmann, D., \& Burton, W. B. 1997, Atlas of galactic neutral hydrogen (Cambridge University Press)

Haslam, C., Salter, C., Stoffel, H., \& Wilson, W. 1982, A\&AS, 47,1

Houck, J., \& Bregman, J. 1990, ApJ, 352, 506

Hurwitz, M. 1994, ApJ, 433, 149

Indebetouw, R., \& Shull, J. M. 2004a, ApJ, 605, 205

Indebetouw, R., \& Shull, J. M. 2004b, ApJ, 607, 309

Ito, M., \& Ikeuchi, S. 1988, PASJ, 40,403

Jenkins, E., \& Tripp, T. 2001, ApJS, 137, 297

Korpela, E., Edelstein, J., Kregenow, J., et al. 2006, ApJ, 644, L163

Kregenow, J., Edelstein, J., Korpela, E., et al. 2006, ApJ, 644, L167

Lallement, R., Welsh, B. Y., Vergely, J. L., et al. 2003, A\&A, 411, 447

Martin, C., \& Bowyer, S. 1990, ApJ, 350, 242

McCammon, D., Almy, R., Apodaca, E., et al. 2002, ApJ, 576, 188

Murthy, J., \& Shanow, D. 2004, ApJ, 615, 315

Raymond, J., Blair, W., Long, K., et al. 1997, ApJ, 482, 881

Sankrit, R., Blair, W., \& Raymond, J., ApJ, 589, 242

Savage, B., Sembach, K., \& Lu, L. 1997, AJ, 113, 2158

Savage, B., Sembach, K., Wakker, B., et al. 2003, ApJS, 146, 125

Schlegel, D., Finkbeiner, D., \& Davis, M. 1998, ApJ, 500, 525

Sembach, K., \& Savage, B. D. 1992, ApJS, 83, 147

Sembach, K., Savage, B. D., \& Tripp, T. M. 1997, ApJ, 480, 216

Sembach, K., Wakker, B., Savage, B., et al. 2003, ApJS, 146, 165

Shapiro, P. R., \& Field, G. B. 1976, ApJ, 205, 762

Shelton, R. L. 1998, ApJ, 504, 785

Shelton, R., Kruk, J., Murphy, E., et al., 2001, ApJ, 560, 730

Shelton, R., Sallmen, S., \& Jenkins, E. 2007, ApJ, 659, 365

Shinn, J.-H., Min, K. W., Lee, C.-N., et al. 2006, ApJ, 644, L189

Shull, J. M., \& Slavin, J. 1994, ApJ, 427, 784

Slavin, J., Shull, J. M., \& Begelman, M. 1993, ApJ, 407, 83

Snowden, S., Egger, R., Freyberg, M., et al. 1997, ApJ, 485, 125

Willingale, R., Hands, A., Warwick, R., Snowden, S., \& Burrows, D. 2003, MNRAS, 343, 995

Young, P. R., Del Zanna, G., Landi, E., et al. 2003, ApJS, 144, 135

Zsargo, J., Sembach, K., Howk, J., \& Savage, B. 2003, ApJ, 586, 1019 